\title{
Quantification of the myocardial partition coefficient for intravenous iron (ferric carboxymaltose) using T1 mapping cardiovascular magnetic resonance
}

\author{
Goran Abdula2*, Arman Valadkhani ${ }^{1}$, Magnus Lundin², Peder Sörensson ${ }^{1}$, Andreas Sigfridsson ${ }^{2}$, Martin Ugander ${ }^{2}$ \\ From 19th Annual SCMR Scientific Sessions \\ Los Angeles, CA, USA. 27-30 January 2016
}

\section{Background}

Cardiovascular magnetic resonance (CMR) T1 mapping has recently been shown to be highly sensitive for detecting myocardial iron overload. Furthermore, T1-mapping can be used to quantify relative concentrations of contrast agents that shorten $\mathrm{T} 1$. We hypothesized that $\mathrm{T} 1$ mapping can detect and quantify the myocardial distribution of a clinically available intravenous iron substitution agent.

\section{Methods}

CMR imaging was performed in healthy male volunteers $(\mathrm{n}=8$, mean \pm SD age $27 \pm 3$ years $)$. T1 of blood and myocardium was quantified using a modified Look-Locker inversion recovery (MOLLI) sequence for T1-mapping in a mid-ventricular short-axis slice at 1.5T (Siemens Aera). Images were acquired before and at regular intervals up to 50 minutes after onset of a 15 minute long injection of $20 \mathrm{ml}(50 \mathrm{mg}$ iron/ml) ferric carboxymaltose (Vifor Pharma). T1, R1 and $\Delta \mathrm{R} 1$ of myocardium and blood, and the partition coefficient (lambda) for myocardium were measured over time.

\section{Results}

Both myocardial and blood T1 were shortened after intravenous injection of ferric carboxymaltose (Figure 1). Thus, there is an increase in both R1, which is the reciprocal of $\mathrm{T} 1$, and $\Delta \mathrm{R} 1$, which is the difference between postcontrast and pre-contrast $\mathrm{R} 1$, and is linearly related to contrast concentration. Notably, lambda, which is the ratio

\footnotetext{
${ }^{2}$ Department of clinical physiology, Karolinska University Hospital, Karolinska Institute, Stockholm, Sweden

Full list of author information is available at the end of the article
}

of $\Delta \mathrm{R} 1$ of myocardium to $\Delta \mathrm{R} 1$ of blood, remains constant over time (mean $\pm \mathrm{SEM}, 61 \pm 6 \%$ at 30 minutes).

\section{Conclusions}

T1 mapping can be used to detect and quantify the myocardial distribution of ferric carboxymaltose (figure 2). Furthermore, since lambda remains unchanged over time, the concentration of ferric carboxymaltose in normal myocardium is in a dynamic equilibrium with the blood pool 20-50 minutes after intravenous injection. However lambda in healthy myocardium for ferric carboxymaltose ( $60 \%)$ was considerably higher than lambda for gadolinium-based extracellular contrast agents from the literature $(\sim 40-45 \%)^{1}$, thus indicating that ferric carboxymaltose distributes to a greater extent into the myocardium than extracellular agents, most likely by distribution also into the intracellular space. The described technique opens up the new possibility for using CMR to study in vivo myocardial iron physiology in health and disease.

\section{Authors' details \\ 'Department of cardiology, Karolinska University Hospital, Karolinska Institute, Stockholm, Sweden. ${ }^{2}$ Department of clinical physiology, Karolinska University Hospital, Karolinska Institute, Stockholm, Sweden.}

Published: 27 January 2016

doi:10.1186/1532-429X-18-S1-P229

Cite this article as: Abdula et al:: Quantification of the myocardial partition coefficient for intravenous iron (ferric carboxymaltose) using

T1 mapping cardiovascular magnetic resonance. Journal of Cardiovascular Magnetic Resonance 2016 18(Suppl 1):P229. 

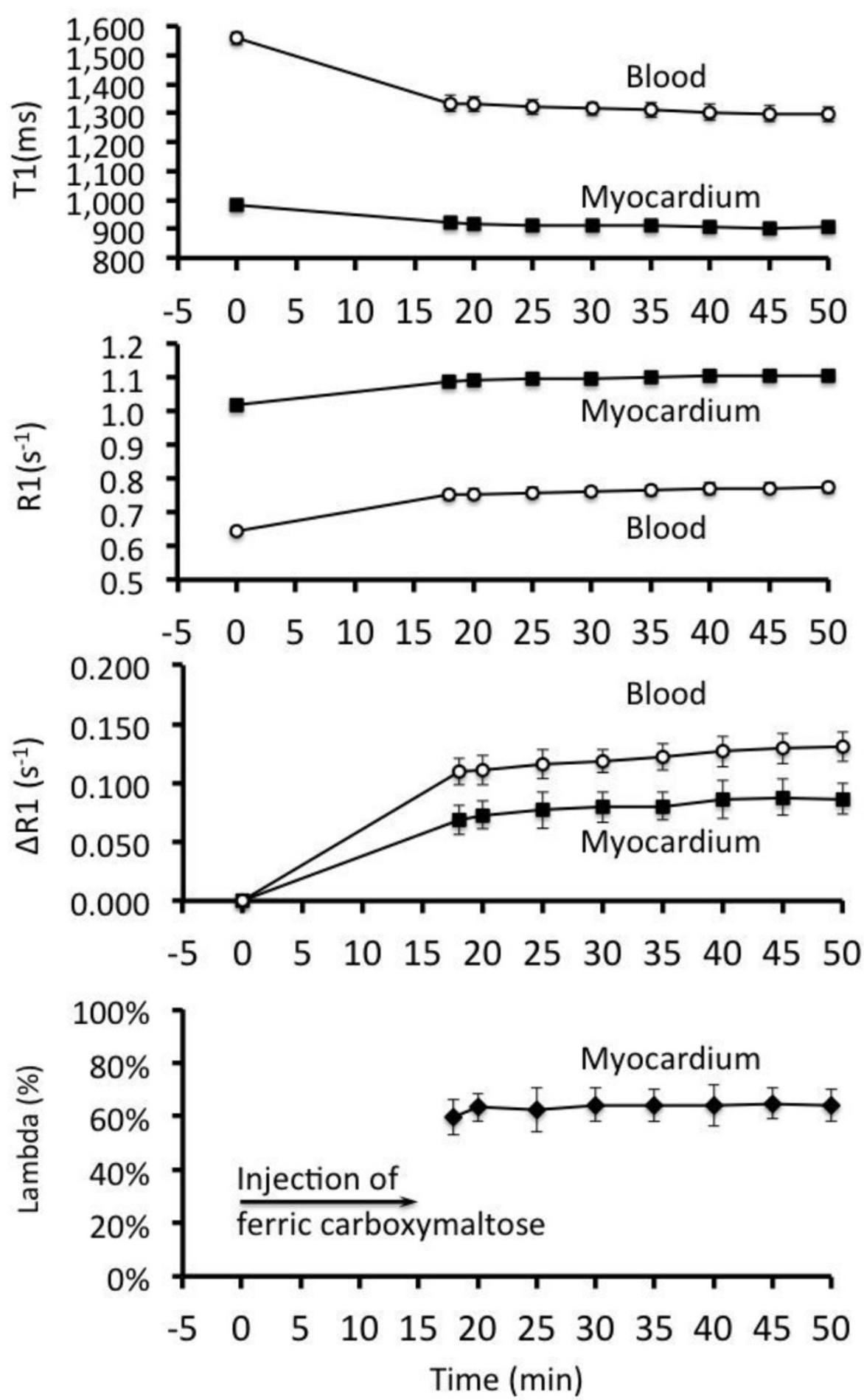

Figure 1 Mean values $( \pm$ SEM) for myocardial (black squares) and blood (white circles) T1, R1, $\Delta R 1$ and partition coefficient (lambda) over time after injection of ferric carboxymaltose in healthy volunteers $(\mathbf{n}=\mathbf{8})$. R1 is equal to $1 / T 1$. $\Delta R 1$ is the difference between postcontrast and pre-contrast R1 and is proportional to contrast concentration. Lambda is the ratio of $\triangle R 1$ of the myocardium to $\Delta R 1$ of the blood. Note that lambda is effectively unchanged between 20-50 minutes after injection. 


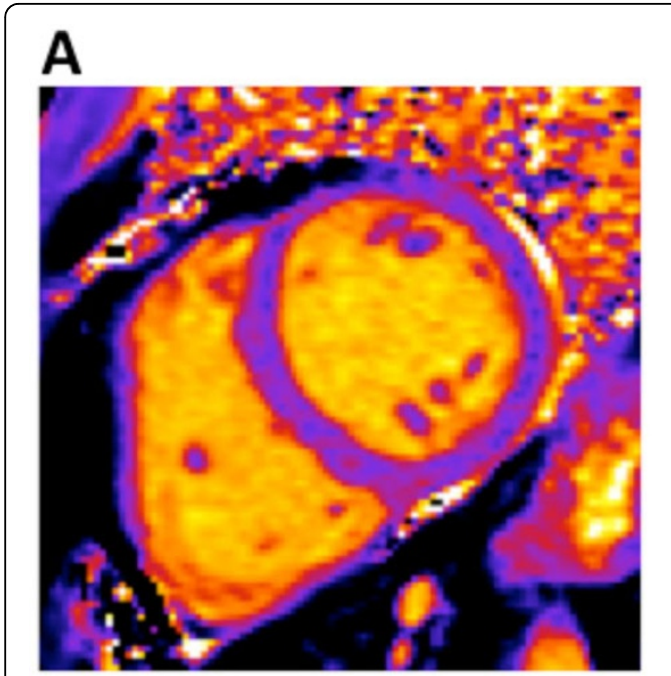

\section{B}
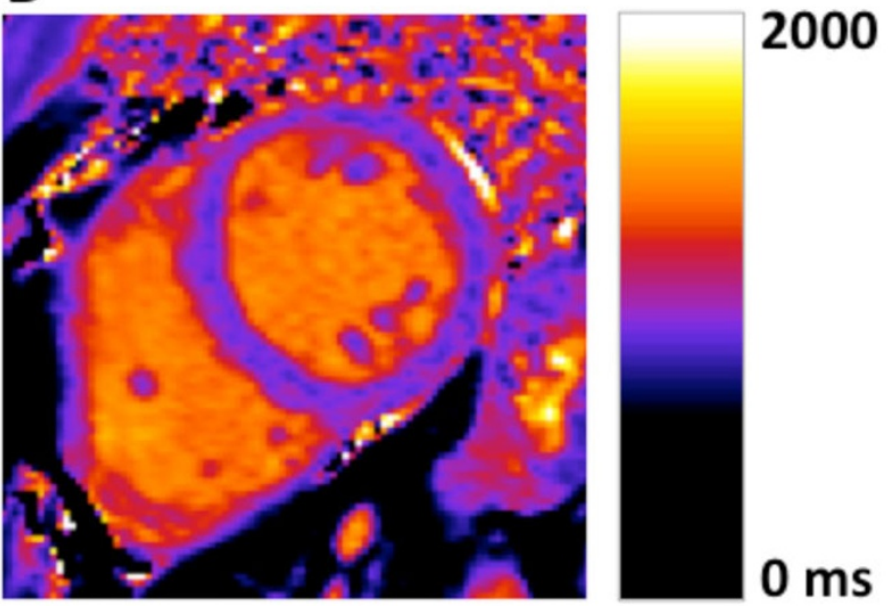

Figure 2 (A) Pre-contrast (native T1) and (B) post-contrast (25 minutes after onset of ferric carboxymaltose injection) short-axis Modified Look-Locker Inversion recovery (MOLLI) sequence in a mid-ventricular slice. Note how T1 is visibly shorter following injection of ferric carboxymaltose compared to native $\mathrm{T} 1$ 